\title{
Efectos de la Jornada Escolar Completa sobre el aprendizaje de los
} estudiantes

Effects of the All-Day Schooling on student learning

Recepción del artículo: 27-10-2020 ～Aceptación del artículo: 13-03-2021

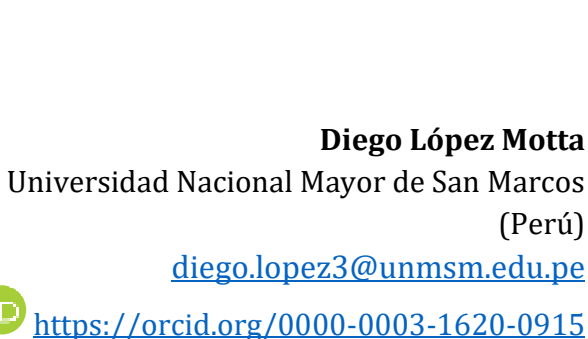

https://orcid.org/0000-0003-1620-0915

\section{Resumen}

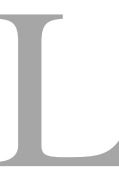

a Jornada Escolar Completa (JEC) apunta a mejorar los aprendizajes de los adolescentes peruanos que cursan el nivel secundario en los colegios públicos. El objetivo del artículo es dar cuenta de los efectos de este modelo educativo en dos escuelas de Lima Metropolitana. El diseño es cualitativo y transversal, por lo que se realizó una descripción etnográfica en simultáneo de una institución educativa en San Juan de Miraflores y de una institución educativa emblemática en Surquillo. Asimismo, se desarrollaron entrevistas a cinco estudiantes de 5 to grado de educación secundaria y observación participante en las aulas. Se evidenció que los avances y desafíos de la JEC dependen del contexto de los centros educativos y de las demandas de los estudiantes como parte de sus distintas apuestas profesionales. La diferencia más resaltante fue que los participantes del estudio en el colegio de San Juan de Miraflores tienen una percepción positiva de la JEC, mientras que los del colegio de Surquillo califican como insuficiente la formación en áreas curriculares como Matemática, que precisamente con la JEC ha tenido un incremento en las horas lectivas y una transformación de la modalidad de enseñanza.

Palabras Clave: Jornada Escolar Completa, política educacional, fin de la escolaridad, formación profesional, adolescencia.
López-Motta, D. (2021). Efectos de la Jornada Escolar Completa sobre el aprendizaje de los estudiantes. Revista ConCiencia EPG, 6(2), 30-52. https://doi.org/10.32654/CONCIENCIAEP 
Abstract

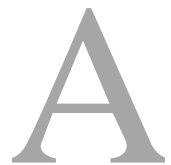

ll-Day Schooling aims to improve the learning of Peruvian teenagers who attend the secondary level at the public schools. The objective of the article is to give an account of the effects of this educational model in two schools in Metropolitan Lima. The design is qualitative and transversal, so an ethnographic description was made simultaneously of an educational institution in San Juan de Miraflores and an emblematic educational institution in Surquillo. In addition, in-depth interviews were conducted with five fifth grade secondary students and participant observation in the classrooms. It was evident that the advances and challenges of All-Day Schooling depend on the context of the educational centers and the demands of the students as part of their different professional bets. The most outstanding difference was that the participants of the study in the school of San Juan de Miraflores have a positive perception of All-Day Schooling, while those of the school of Surquillo qualify as insufficient the formation in curricular areas such as Mathematics, which precisely with All-Day Schooling has had an increase in the school hours and a transformation of the teaching modality.

Key Words: All-Day Schooling, educational policy, school leaving, vocational training, adolescence.

\section{Introducción}

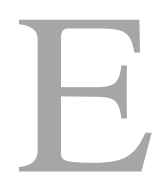

n el marco de la Jornada Escolar Completa (JEC), la presente investigación trata de analizar los efectos de esta política educativa sobre el aprendizaje de los estudiantes. Desde 2015, en una selección de escuelas públicas peruanas, funciona la JEC, que incrementa de 35 a 45 las horas lectivas semanales en el nivel secundario, distribuidas sustancialmente en las áreas curriculares de Comunicación, Matemática, Inglés y Educación para el Trabajo (EPT). Sus objetivos generales son mejorar la calidad de la educación secundaria y promover el cierre de brechas en el Perú.

La JEC ha tenido como efecto incrementar las aspiraciones de los escolares de cursar estudios superiores universitarios (Sánchez y Favara, 2019). Sin embargo, es pertinente evidenciar si su componente pedagógico les ofrece concretamente las herramientas para insertarse a la educación universitaria, la cual es un anhelo tanto para ellos como para sus familias y docentes (Guerrero, 2014). En este sentido, se encontrará una brecha entre los contenidos curriculares de Matemática, curso que con la JEC ha aumentado sus horas lectivas semanales, y las aspiraciones de los participantes del estudio, quienes afirman que la enseñanza de esta materia no contribuye a aprobar un examen de admisión.

Para analizar los efectos de la JEC sobre el aprendizaje de los estudiantes, se presenta una aproximación principalmente etnográfica, la cual construye conocimiento a partir de los testimonios de los actores 
sociales y la observación participante (Guber, 2001; Serra, 2004; Álvarez, 2008; 2011; Restrepo, 2016). De esta manera, los hallazgos son producto de entrevistas con escolares de 5to grado de educación secundaria y de observaciones de aula. Además, un estudio etnográfico permite registrar el cambio desde la experiencia (Marcus y Fischer, 1986), en este caso de estudiantes que se han adaptado a la JEC reconociendo sus avances y desafíos.

Desde las ciencias sociales en el Perú, existe una amplia literatura que articula educación con antropología (Portocarrero y Oliart, 1989; Benavides et al., 2006; Montero et al., 2009; Ames et al. 2010; Ames y Rojas, 2011; Oliart, 2011; Eguren y de Belaunde, 2012; González et al., 2017). Sin embargo, es importante mencionar que el presente artículo se desmarca de esas entradas analíticas, puesto que busca evidenciar cómo una política educativa como la JEC tiene efectos distintos según el tipo de colegio.

Cabe resaltar que la antropología se ha aproximado a los proyectos de desarrollo humano, que para efectos de este estudio cobran forma de diseños pedagógicos como lo es la JEC. En línea con Appadurai (2004), estas intervenciones deben poner en práctica una serie de recursos para identificar las aspiraciones de los actores sociales. Así, un proyecto educativo como la JEC supondría incluir los anhelos futuros de los alumnos, como la aprobación de un examen de admisión y el ingreso a la universidad. No obstante, en la sección de resultados se evidenciará cómo la demanda de una escolar por mejor preparación académica no corresponde a uno de los objetivos de la JEC, que es precisamente responder a las necesidades y a los intereses de los estudiantes.

A raíz de la ejecución de la JEC, algunas de las investigaciones se centran en evaluaciones de su implementación. Entre los principales hallazgos se identifica que, por un lado, en 5to grado de educación secundaria el componente pedagógico es el que presenta más complicaciones para los alumnos (Alcázar, 2016) y, por otro lado, entre los estudiantes de 2do grado de educación secundaria mejoraron los niveles de aprendizaje de Matemática (Agüero, 2016).

Otros estudios han examinado el nivel de conocimiento de los directores de colegios de Puno sobre la JEC (Alanoca, 2016), el estrés académico de alumnos de 5 to grado de educación secundaria que estudian bajo el mencionado modelo educativo en Cajamarca (Rojas y Ramos, 2017) y la conformidad de una mayoría de docentes en Arequipa con el clima organizacional de la JEC (Feliciano, 2017). Así, se evidencia que los efectos de esta política son heterogéneos según las distintas instancias de la comunidad educativa, como directivos, alumnado $\mathrm{y}$ plana docente.

También se han explorado el malestar de un grupo de padres de familia en Puno con el componente pedagógico de la JEC porque no perciben cambios en los aprendizajes de sus hijos (Mamani, 2017), las consecuencias en la economía familiar de estudiantes de Huancavelica porque tienen menos tiempo en el campo (Gantu, 2018), la aprobación de las normas de 
ejecución de la JEC por parte del personal (Ccopa, 2018) y la eficiencia de los acompañantes pedagógicos en centros educativos de Puno (Yana y Adco, 2018).

Por su parte, los trabajos comparativos han profundizado en el aprendizaje de los adolescentes. Peralta (2018) encuentra que, en Puno, el logro en el aprendizaje de Inglés de alumnos de 3er grado de educación secundaria es ligeramente superior en un colegio con la Jornada Escolar Regular (JER) en contraste con uno con la JEC. En Madre de Dios, Villegas (2019) observa que el nivel satisfactorio de lectura es mayor en estudiantes de 2 do grado de educación secundaria en una escuela con la JER y menor en una con la JEC.

Como se ha visto, la JEC en el Perú ha suscitado una cantidad considerable de investigaciones en este breve tiempo de ejecución. A nivel internacional, el Banco Mundial (2016) muestra que la transición a escuelas de tiempo completo ha reducido el abandono escolar y mejorado los resultados en las pruebas estandarizadas. En Chile y Brasil, se han demostrado efectos positivos en la retención, mientras que en Argentina aumentaron las tasas de graduación. Del mismo modo, en Guatemala, Honduras, Nicaragua, El Salvador y República Dominicana se implementaron las escuelas de tiempo completo en el nivel secundario a fin de reducir el abandono escolar.

Para el caso chileno, Contreras et al. (2018) identifican que la extensión horaria de la jornada escolar es uno de los factores que influyen en las decisiones de los adolescentes en su transición de la escuela al trabajo. En Colombia, entre varias iniciativas para favorecer el acceso de los jóvenes a la educación superior y al mercado laboral, se extendió la jornada escolar de 5 a 8 horas diarias (Bernal et al., 2018).

El objetivo del presente estudio es mostrar cómo la JEC es asimilada de manera distinta por un grupo de estudiantes de una institución educativa en San Juan de Miraflores (SJM) y por otro grupo de una institución educativa emblemática en Surquillo. El artículo incluye cuatro secciones. En la primera, se describe el método empleado, el cual abarca a los participantes, los instrumentos y el procedimiento. La segunda sección presenta los resultados obtenidos como producto del trabajo de campo y en la tercera se expone la discusión. Por último, el cuarto acápite plantea las conclusiones del estudio y enuncia algunas sugerencias para la formulación de políticas educativas.

\section{Método}

La pregunta que se busca responder es: ¿Cuáles son los efectos de la JEC sobre el aprendizaje de los estudiantes de un colegio de SJM y otro de Surquillo? A fin de obtener las percepciones de los estudiantes, se adoptó principalmente una metodología cualitativa, utilizando como técnicas las entrevistas y la observación participante. De acuerdo con Ames, Rojas y Portugal (2010), un abordaje de este tipo es el más pertinente para realizar estudios con niños $\mathrm{y}$ adolescentes.

En línea con Restrepo (2016), lo crucial en un estudio etnográfico son tanto 
las prácticas como los significados que estas adquieren para las personas que las realizan. Además, destaca que la escritura etnográfica se construye en base a las observaciones y conversaciones sostenidas durante el trabajo de campo. En este sentido, el presente estudio emplea un diseño etnográfico, en el que la presencia en el campo y la vinculación con los participantes permiten establecer una relación que contribuye a la obtención de datos y de información fiable (Serra, 2004).

El estudio es transversal en la medida que registra el comportamiento y los discursos de adolescentes de dos distritos de la ciudad de Lima en un momento determinado: el año escolar 2018. Cabe precisar que otras investigaciones de corte longitudinal siguen en el tiempo a los actores sociales para evidenciar la reconfiguración de sus aspiraciones en el futuro. La validez de un análisis transversal como el que se presenta consiste en que el objeto del estudio no son los anhelos de los estudiantes de 5to grado de educación secundaria al finalizar el período escolar, sino describir los efectos de una política educativa como la JEC en un momento determinado.

Participantes: están conformados por cinco estudiantes hombres y mujeres, dos por la escuela de Surquillo y tres por la de SJM, que en 2018 cursaron 5to grado de educación secundaria. Las edades están comprendidas entre los 15 y 18 años. Esta muestra pertenece a los distritos de SJM, Villa María del Triunfo (VMT), Villa El Salvador (VES) y Surquillo, ubicados en Lima Sur.
El tipo de muestreo es intencional, pues permite al investigador decidir los elementos que integrarán la muestra según los objetivos del estudio y sus percepciones. Asimismo, se aplicó un muestreo de informante clave, a través del cual "se escogen personas por razones especiales como: conocimiento del tema, experiencia o haber sido protagonista del fenómeno a estudiar, grado de participación en el grupo social y en la toma de decisiones y su disposición para brindar información, entre otros" (López, 2004, p.74).

Considerando lo anterior, el criterio de inclusión en la selección de la muestra fue el nivel adecuado de participación y atención que los cinco estudiantes entrevistados mostraron en las distintas materias. Esta decisión se respalda por la literatura: al término del nivel secundario, un correcto rendimiento escolar se manifestaría en un futuro éxito tanto en el campo académico como en el profesional (Rojas y Cussianovich, 2013) y la etapa escolar influye en las decisiones futuras de los adolescentes (Herrera, Lagrou y Lens, 2002). En este sentido, sus apreciaciones iban a resultar relevantes para confirmar o no si la JEC cumplía un rol sustancial en su formación.

Además, se entrevistó a la docente de Matemática de la escuela de Surquillo. Esta materia tuvo un incremento en las horas lectivas y una transformación en su metodología de enseñanza tras la implementación de la JEC, por lo que el testimonio de la profesora es un elemento de primer orden para el análisis de esa política educativa y su impacto en el aprendizaje de los estudiantes. Conforme 
iba avanzando el trabajo de campo y se detectó que la modalidad pedagógica de Matemática ocasionaba problemas entre los participantes del estudio en el colegio de Surquillo y ventajas para los de la escuela de SJM, se excluyó de la investigación a los docentes de las otras áreas curriculares.

La sección del centro educativo de Surquillo en la que se realizó observación participante cuenta con 21 estudiantes, mientras que el aula del colegio de SJM cuenta con 25. Por ello, el criterio de exclusión del resto de alumnos se basó en su desempeño, que fue registrado durante las clases, el cual no era tan participativo. Inicialmente, se entrevistaron a más escolares que la muestra seleccionada, pero sus testimonios se excluyeron del estudio porque podían alterar la naturaleza comparativa de la recepción de Matemática en ambos colegios.

Si bien otros estudios han analizado la JEC en 2do grado de educación secundaria (Agüero, 2016; Villegas, 2019) y en 3ro (Peralta, 2018), en el presente artículo se excluyeron estos grados y se trabajó solo con 5to porque en este nivel el escolar está en una edad en la que puede reflexionar más sobre su futuro, adquirir una conciencia crítica sobre su situación personal y evaluar los beneficios de la educación superior (Post, 1987).

Instrumentos: en tanto el estudio representa una aproximación antropológica a una política educativa como la JEC, los materiales utilizados fueron la observación participante y las entrevistas.
Observación participante: durante el trabajo de campo, la permanencia en el salón de clases contribuyó a entender cómo las y los estudiantes asimilan su formación escolar. Según Restrepo (2016), el terreno es el lugar donde se efectúa el trabajo de campo y es la unidad de observación desde donde se aborda el problema a estudiar. La presencia del investigador en el aula prestando atención a la clase y a todo lo que acontecía, y tomando apuntes, se llevó a cabo porque "es imposible estudiar a un grupo sin ser parte de él" (Guber, 2001, p. 72). Como material de registro de información, la observación participante permite un conocimiento real del objeto porque no abstrae ni cuantifica, sino que recoge todos los aspectos de los fenómenos estudiados (Alvira, 1983).

Para llevar a cabo la observación participante en las aulas, se utilizó un diario de campo, que en estudios cualitativos "es el instrumento de registro de datos del investigador de campo, donde se anotan las observaciones (notas de campo) de forma completa, precisa y detallada" (Ruano, 2007, p. 3). En esa línea, durante los meses de trabajo, se apuntaron las fechas y los cursos de observación, así como algunas incidencias en las aulas relacionadas con la JEC.

Durante el período de observaciones, se registró el desempeño académico de las y los estudiantes en las clases y en base a eso se definió la muestra para entrevistar. Según Ames y Rojas (2011), la descripción del espacio escolar contribuye a contextualizar las percepciones de los estudiantes. En este sentido, fue necesario 
combinar la presencia en el campo con las entrevistas.

Entrevistas: su aplicación se llevó a cabo de manera individual después de tres meses de observación participante en las aulas. Se procedió de este modo para que, al iniciar los diálogos, los estudiantes ya estuvieran familiarizados, en la medida de lo posible, con la presencia del investigador en su entorno escolar.

Para el estudio se empleó la entrevista semiestructurada, que permitió enfatizar en las temáticas principales relacionadas a la JEC y facilitó la opción de repreguntas. Para Blasco y Otero (2008), en este tipo de entrevista las preguntas están definidas previamente por el investigador, pero el orden puede variar en función del participante entrevistado. Si bien las preguntas se prepararon con anticipación, la entrevista semiestructurada generó que las y los adolescentes se explayaran sobre otros temas más allá del ámbito escolar, como personales y familiares. Antes de iniciar las entrevistas, se explicaron los objetivos del estudio a los participantes y se resolvieron sus preguntas al respecto.

La voz de los estudiantes constituye la principal materia prima para el análisis. Como sugiere Ibáñez (1986, p. 44), las preguntas y las respuestas se dieron en un lenguaje no solo "comprensible por todos", sino "igualmente comprensible por todos", sin caer en contradicciones ni ambigüedades.

Desde una interpretación antropológica de la educación, algunos estudios en el Perú como los repasados
(Ames et al., 2010; Ames y Rojas, 2011) proponen la importancia de una metodología cualitativa para aproximarse al análisis del sistema escolar. Por ello, los instrumentos empleados en la presente investigación etnográfica tienen validez por dar cuenta de los efectos de la JEC en el aprendizaje de los estudiantes de 5to grado de educación secundaria a través de la observación participante y las entrevistas. Conocer la realidad implica una interpretación adecuada al nivel del sentido de los propios actores sociales (Alvira, 1983) y, en esa línea, tanto el diario de campo como la entrevista semiestructurada permitieron registrar el significado que un grupo de escolares le da a una política educativa.

Procedimiento: cuando se inició el trabajo de campo en ambos centros educativos, se comunicaron los objetivos de la investigación a los estudiantes de las aulas seleccionadas. Luego de los primeros 3 meses de observación participante, se seleccionó la muestra para las entrevistas y se obtuvo el consentimiento informado de los adolescentes, a quienes se les pidió que salieran de sus aulas un momento para explicarles por qué fueron seleccionados y aclararles la naturaleza del estudio. En todo momento, los padres estuvieron al tanto de la investigación realizada. Haber efectuado un estudio con menores de edad implicó un tratamiento ético de la información y el cuidado de los participantes.

Por otra parte, el trabajo de campo también contó con el consentimiento informado de las autoridades escolares, desde los directivos hasta los docentes y tutores. Se procedió de este modo porque se 
suele recomendar un ambiente de confianza y diálogo horizontal para el trabajo con adolescentes (Ames et al., 2010).

Respecto a la protección de los participantes, el colegio de Surquillo estipuló que la totalidad de entrevistas con los estudiantes se desarrollase en la oficina de la Subdirección General. Para tener uniformidad con el trabajo, en el colegio de SJM las entrevistas también se realizaron en una oficina de ese tipo, como la Dirección General. Con el propósito de manejar la confidencialidad, tratándose de menores de edad, no se utilizan los nombres reales de los participantes de la investigación. Se seleccionaron para su interpretación los discursos más representativos del tema en estudio a partir de la escucha de cada audio de las entrevistas

\section{Resultados}

Los resultados son producto de un diseño etnográfico que permite dar cuenta de cómo los actores sociales realizan sus propias interpretaciones y reelaboran los significados de una política educativa como la JEC. Este enfoque, que utiliza para el análisis la observación participante y los testimonios de dos grupos de estudiantes, evidenció que la JEC no se ha adecuado a sus pretensiones futuras en el colegio de Surquillo y ha generado una valoración positiva de la formación en el colegio de SJM.

La etnografía consiste en la interpretación sobre lo que el investigador percibió desde los testimonios de los sujetos de estudio y la observación participante (Guber, 2001; Serra, 2004; Álvarez, 2008; 2011; Restrepo, 2016). En este sentido, las percepciones de los estudiantes, en función de las entrevistas concretadas en cada colegio, se pueden observar en las siguientes tablas: 
Tabla 1

Percepciones de futuro en el contexto de la JEC en el colegio de Surquillo

\begin{tabular}{|c|c|c|}
\hline Preguntas & Estudiante 1 & Estudiante 2 \\
\hline $\begin{array}{l}\text { ¿Qué carrera quisieras } \\
\text { estudiar y por qué? }\end{array}$ & $\begin{array}{l}\text { Ingeniería Industrial, } \\
\text { Psicología o Educación. } \\
\text { Ingeniería Industrial es } \\
\text { como ir a diferentes } \\
\text { empresas y verificar. Algo } \\
\text { así he escuchado sobre esa } \\
\text { carrera. Psicología para } \\
\text { poder dar consejos, yo } \\
\text { también soy amigable con } \\
\text { las personas y me cuentan. } \\
\text { Educación porque me } \\
\text { gustaría ser profesora de } \\
\text { inglés. Me gusta inglés. }\end{array}$ & $\begin{array}{l}\text { Derecho porque me gusta } \\
\text { mucho el curso de Cívica. } \\
\text { Me llama bastante la } \\
\text { atención. Y Psicología } \\
\text { porque me gustaría } \\
\text { entender y ayudar a las } \\
\text { demás personas. Sé que } \\
\text { algunas personas tienen } \\
\text { problemas y me gustaría } \\
\text { escucharlas y ayudarlas. }\end{array}$ \\
\hline $\begin{array}{l}\text { ¿Crees que este colegio con } \\
\text { Jornada Escolar Completa } \\
\text { te está preparando como } \\
\text { debe ser para la } \\
\text { universidad? }\end{array}$ & $\begin{array}{l}\text { No tanto porque en } \\
\text { Matemática debería haber } \\
\text { un poco más de temas } \\
\text { porque cada año a veces se } \\
\text { repite el mismo tema. Eso } \\
\text { es lo que me he dado } \\
\text { cuenta. Falta todavía un } \\
\text { poco. }\end{array}$ & $\begin{array}{l}\text { Sí, pero siento que en } \\
\text { Matemática todavía } \\
\text { estamos en lo básico, me } \\
\text { gustaría aprender un poco } \\
\text { más. Siento que nos } \\
\text { enseñan muy poco para el } \\
\text { examen de admisión de } \\
\text { alguna universidad. Aún } \\
\text { estamos en Pitágoras y me } \\
\text { gustaría que enseñen un } \\
\text { poquito más porque en } \\
\text { esos test de universidad } \\
\text { que yo he visto por internet } \\
\text { es un poquito más difícil } \\
\text { matemática y siento que es } \\
\text { muy poco lo que enseñan } \\
\text { aquí. }\end{array}$ \\
\hline $\begin{array}{l}\text { Aparte de la universidad, } \\
\text { ¿qué otra alternativa tienes } \\
\text { acabando el colegio? }\end{array}$ & $\begin{array}{l}\text { Trabajar por un tiempo y } \\
\text { de ahí también prepararme } \\
\text { en algo que me guste. }\end{array}$ & $\begin{array}{l}\text { El otro año mi mamá me } \\
\text { quería meter al Icpna para } \\
\text { aprender inglés, pero de ahí } \\
\text { nada. Ninguna academia } \\
\text { que tenga en mente. }\end{array}$ \\
\hline
\end{tabular}


Tabla 2

Percepciones de futuro en el contexto de la JEC en el colegio de SJM

\begin{tabular}{|c|c|c|c|}
\hline Preguntas & Estudiante 1 & Estudiante 2 & Estudiante 3 \\
\hline $\begin{array}{l}\text { ¿Qué carrera } \\
\text { quisieras estudiar } \\
\text { y por qué? }\end{array}$ & $\begin{array}{l}\text { Diseño Gráfico o } \\
\text { Dibujo Artístico. } \\
\text { Uno de los dos, } \\
\text { porque sé dibujar y } \\
\text { sé combinar los } \\
\text { colores. }\end{array}$ & $\begin{array}{l}\text { Administración de } \\
\text { Empresas porque } \\
\text { me gustan los } \\
\text { números y quisiera } \\
\text { cambiar otro tipo } \\
\text { de carrera en el } \\
\text { ambiente de mi } \\
\text { familia porque } \\
\text { tengo mi hermano } \\
\text { mayor que es } \\
\text { doctor, estudió } \\
\text { Medicina. Yo } \\
\text { quisiera cambiar } \\
\text { otro ambiente de } \\
\text { carrera. }\end{array}$ & $\begin{array}{l}\text { Psicología porque } \\
\text { me gusta el curso } \\
\text { de Persona, puedo } \\
\text { expresarme bien, } \\
\text { me gustan los } \\
\text { temas que tiene esa } \\
\text { carrera y todo eso. } \\
\text { Y los campos } \\
\text { laborales, que más } \\
\text { que nada son en } \\
\text { clínicas, en penales, } \\
\text { en los colegios. Me } \\
\text { gusta dialogar con } \\
\text { las personas y dar } \\
\text { consejos. }\end{array}$ \\
\hline $\begin{array}{l}\text { ¿Crees que este } \\
\text { colegio con } \\
\text { Jornada Escolar } \\
\text { Completa te está } \\
\text { preparando como } \\
\text { debe ser para la } \\
\text { universidad? }\end{array}$ & $\begin{array}{l}\text { En algunos será, } \\
\text { pero en otros no. En } \\
\text { los nuevos que han } \\
\text { ingresado, sí, como } \\
\text { el profesor de } \\
\text { Matemática. Pero en } \\
\text { los demás, en la } \\
\text { mayoría de } \\
\text { profesores, no. Por } \\
\text { ejemplo, el profesor } \\
\text { de Arte, que no } \\
\text { enseña casi nada. Y } \\
\text { el profesor de } \\
\text { Matemática, que es } \\
\text { nuevo, pero él sí } \\
\text { enseña. Sí enseña } \\
\text { bien, pero el } \\
\text { profesor de Arte no. } \\
\text { Como lo que tiene } \\
\text { que ser el arte, no } \\
\text { En lo que se trata de } \\
\text { artística y pintura, } \\
\text { no enseña nada. }\end{array}$ & $\begin{array}{l}\text { Creo que estos } \\
\text { últimos años me } \\
\text { está preparando } \\
\text { mejor que toda la } \\
\text { secundaria que he } \\
\text { estudiado aquí. } \\
\text { Ahora en 5to ha } \\
\text { mejorado más la } \\
\text { educación. En la } \\
\text { materia de } \\
\text { Matemática han } \\
\text { traído a un } \\
\text { profesor que ahora } \\
\text { nos ha hecho } \\
\text { renovar más el } \\
\text { aprendizaje. Nos } \\
\text { está enseñando } \\
\text { cosas nuevas que } \\
\text { no habíamos } \\
\text { aprendido desde } \\
\text { 1ro de secundaria. }\end{array}$ & $\begin{array}{l}\text { Esta institución } \\
\text { como que sus } \\
\text { enseñanzas son } \\
\text { más largas, nos } \\
\text { brindan más horas, } \\
\text { es diferente a mi } \\
\text { otro colegio. Y sí, } \\
\text { tocan nuevos } \\
\text { temas, te explican } \\
\text { bien, te enseñan } \\
\text { bien. Y sí, creo que } \\
\text { sí es una buena } \\
\text { institución } \\
\text { educativa porque } \\
\text { mi otro colegio era } \\
\text { medio relajado, no } \\
\text { les hacían caso a los } \\
\text { profesores. En } \\
\text { cambio, acá es un } \\
\text { poco más recto. }\end{array}$ \\
\hline
\end{tabular}




\begin{tabular}{|l|l|l|l|}
\hline $\begin{array}{l}\text { Aparte de la } \\
\text { universidad, ¿qué } \\
\text { otra alternativa } \\
\text { tienes acabando el } \\
\text { colegio? }\end{array}$ & $\begin{array}{l}\text { Estudiar y trabajar. } \\
\text { Por ahora cuando } \\
\text { termine secundaria } \\
\text { voy a ayudar a mi } \\
\text { padrastro mientras } \\
\text { tanto. Y cuando ya } \\
\text { sea mayor de edad } \\
\text { ahí sí pienso buscar } \\
\text { mi trabajo. }\end{array}$ & $\begin{array}{l}\text { Trabajar saliendo } \\
\text { de la escuela para } \\
\text { apoyar en mi } \\
\text { carrera que quiero } \\
\text { estudiar para no } \\
\text { cargarle tanto peso } \\
\text { a mis padres. }\end{array}$ & $\begin{array}{l}\text { Estudiar una } \\
\text { carrera técnica que } \\
\text { es Cosmetología } \\
\text { para hacer } \\
\text { peinados. Como la } \\
\text { carrera es de tres } \\
\text { meses, estudiar y } \\
\text { tener un trabajo } \\
\text { fijo. Y con ese } \\
\text { trabajo fijo salir yo } \\
\text { adelante, poder } \\
\text { estudiar alguna } \\
\text { otra carrera o para } \\
\text { ayudarme yo } \\
\text { misma en mi casa o } \\
\text { comprar mis cosas } \\
\text { ya para futuro. }\end{array}$ \\
& & & \\
\end{tabular}

Los resultados de las entrevistas indican que los alumnos de dos colegios con la JEC tienen aspiraciones de seguir estudios universitarios, en concordancia con lo reportado por Sánchez y Favara (2019). Asimismo, dos estudiantes del colegio de Surquillo se mostraron inconformes con la enseñanza de Matemática, curso que tras la implementación de la JEC ha tenido un incremento de las horas lectivas semanales.

Por el contrario, en el colegio de SJM, dos estudiantes reconocieron un progreso en la enseñanza de Matemática, mientras que uno afirmó que la JEC es mejor debido a que los cursos se dictan por más horas. De esta manera, es posible contrastar los resultados obtenidos en una institución educativa emblemática en Surquillo y en una institución educativa en SJM, en el marco de la JEC.

Para cotejar las percepciones de las entrevistadas en la escuela de Surquillo y profundizar en el componente pedagógico de Matemática, se obtuvo el testimonio de la profesora de esa materia. De esta manera, se garantiza la credibilidad de los datos etnográficos por medio de una estrategia de triangulación de sujetos, basada en el contraste de los puntos de vista de los miembros de la comunidad estudiada (Álvarez, 2008). Cabe señalar que en el colegio de SJM no fue posible dialogar con el docente por falta de disponibilidad en su horario laboral y tampoco se realizó observación de aula a ese curso. 
Tabla 3

Testimonio de la profesora de Matemática en el colegio de Surquillo

\begin{tabular}{|l|l|}
\hline Pregunta & Profesora \\
\hline $\begin{array}{l}\text { ¿Cree que la Jornada } \\
\text { Escolar Completa } \\
\text { está preparando a } \\
\text { los alumnos para la } \\
\text { educación superior? }\end{array}$ & $\begin{array}{l}\text { Nosotros como JEC tenemos ciertos contenidos y materiales } \\
\text { para trabajar. Ahora es aprendizaje basado en problemas. Ese } \\
\text { el alumno no sabe leer problemas, entonces ellos no entienden. } \\
\text { Tú puedes resolver diversos campos de la vida cotidiana, pero } \\
\text { está ingresando geometría, trigonometría, álgebra. Un alumno } \\
\text { que no lee problemas no va a poder comprender lo demás. Esos } \\
\text { lineamientos de secundaria que estamos teniendo con JEC van a } \\
\text { encajar cuando la universidad asuma ese nuevo rol, esos nuevos } \\
\text { contenidos. }\end{array}$ \\
\hline
\end{tabular}

Un enfoque etnográfico se sostiene en la observación participante, "porque permite acceder a un tipo de información que, si no, sería muy difícil de recoger, brindando al investigador la oportunidad de recoger dicha información en persona y conocer y vivir los procesos educativos en toda su complejidad" (Álvarez, 2011, p. 277). En base a las observaciones de aula al curso de Matemática, que fueron registradas en el diario de campo, se presentan los siguientes resultados:

Tabla 4

Dinámica de las clases de Matemática en el colegio de Surquillo

\begin{tabular}{|c|c|}
\hline Clases & Observaciones \\
\hline $\begin{array}{l}\text { Fecha: viernes } 18 \text { de mayo } \\
\text { de } 2018 \\
\text { Hora de inicio: 08:00 a.m. } \\
\text { Hora de fin: 09:30 a.m. } \\
\text { Lugar: aula MATE5(7) }\end{array}$ & $\begin{array}{l}\text { La matemática como ciencia exacta se presenta como más } \\
\text { amena. La profesora utiliza materiales, fomenta el juego y } \\
\text { muestra una performance para enseñar mejor. } \\
\text { Los temas trabajados están en diálogo con casos reales. Por } \\
\text { ejemplo, la profesora escribe en la pizarra: "sólidos } \\
\text { geométricos de revolución en nuestra vida diaria”. Su } \\
\text { método es el más didáctico posible. } \\
\text { La profesora dice: “Hay que pensar", “quiero que estén } \\
\text { atentos”, "mirando ahí". } \\
\text { Siempre hay comparaciones con situaciones de la vida } \\
\text { cotidiana. Los problemas matemáticos que los alumnos } \\
\text { deben resolver se basan en hacer cálculos a elementos como } \\
\text { un volcán, una lámpara y las partes de un auto. }\end{array}$ \\
\hline
\end{tabular}




\begin{tabular}{|l|l|}
$\begin{array}{l}\text { Fecha: viernes 1 de junio de } \\
\text { Hora de inicio: 08:00 a.m. }\end{array}$ & $\begin{array}{l}\text { La profesora genera la participación nombrando los } \\
\text { apellidos o nombres de los alumnos. Deja un problema y da } \\
\text { un momento para que ellos continúen solos. Luego pregunta: } \\
\text { “Cuántos llegaron a la solución y cuántos no?”. }\end{array}$ \\
Hora de fin: 09:30 a.m. & $\begin{array}{l}\text { Varios estudiantes salen a la pizarra. Hay una mayor } \\
\text { participación. Se organizan en grupos de dos para resolver } \\
\text { un problema. La profesora promueve que cada alumno dé su } \\
\text { propia respuesta. }\end{array}$ \\
\hline $\begin{array}{l}\text { Fecha: martes } 4 \text { de } \\
\text { diciembre de 2018 }\end{array}$ & $\begin{array}{l}\text { Por medio de un ejercicio práctico con cañas y burbujas se } \\
\text { explica el concepto de simetría axial. El experimento es } \\
\text { presentado por escolares de otra sección de 5to grado de } \\
\text { educación secundaria que fueron invitados a clase por la } \\
\text { profesora. }\end{array}$ \\
Lora de fin: 03:30 p.m. & $\begin{array}{l}\text { Se muestra que la matemática no es ajena a la vida cotidiana } \\
\text { y está presente en cada momento. }\end{array}$ \\
\hline
\end{tabular}

Finalmente, se encontró que, pese a tener aspiraciones educativas concluyendo la fase escolar, los participantes del estudio contemplan caminos alternativos para su futuro, es decir, son conscientes de sus posibilidades materiales y de sus realidades socioeconómicas antes de ingresar a la universidad. Si la JEC aumenta sus anhelos por la educación superior, también es importante considerar las barreras estructurales que los adolescentes afrontan.

\section{Discusión}

Desde la implementación de la JEC en el Perú, se han realizado estudios sobre sus repercusiones en la comunidad educativa (Alcázar, 2016; Agüero, 2016; Alanoca, 2016; Feliciano, 2017; Mamani, 2017; Rojas y Ramos, 2017; Ccopa, 2018; Gantu, 2018; Peralta, 2018; Yana y Adco, 2018; Villegas, 2019). El presente artículo aporta a la literatura a través de una interpretación antropológica de esta política, considerando que "la etnografía es el registro sensible del cambio en el nivel de la experiencia" (Marcus y Fischer, 1986, p. 130).

De esta manera, se construye conocimiento sobre la JEC desde la subjetividad de los estudiantes, lo cual es posible con un diseño etnográfico que no aborda una política pública como algo abstracto, sino que da cuenta de sus efectos en las prácticas cotidianas de sus usuarios. Como parte de este tipo de estudio, también se realizó observación participante, que permitió una experimentación personal de la JEC para presentar los hallazgos con mayor rigurosidad.

En línea con lo expuesto por Herrera et al. (2002), una satisfactoria inserción social de los adolescentes se produce cuando continúan estudios técnicos o universitarios tras la finalización 
del nivel secundario. Por el contrario, cuando no logran ingresar a una institución de formación superior, se considera que el proceso de inserción social no ha tenido éxito.

Al término de la secundaria, un adecuado rendimiento escolar es síntoma de que a los egresados les irá bien en la vida (Rojas y Cussianovich, 2013). De modo similar, los centros educativos y su cultura escolar tienen una influencia en la elección de dominios de vida de los jóvenes (Herrera et al., 2002). Por ello, las y los estudiantes de 5to grado de educación secundaria se preocupan por comprender las materias que están relacionadas con lo que les puede servir en el futuro. Si bien la JEC ha incrementado las horas de Matemática, los resultados mostrados en la tabla 1 indican que genera más desorientación que certezas, sobre todo para la estudiante 2 del colegio de Surquillo, que expresó que no se siente preparada para la educación superior. Para contextualizar su percepción, la tabla 4 presentó las dinámicas de las clases de ese curso, en las que la metodología no parece ser la que espera una adolescente a punto de egresar del colegio.

Si bien se registraron las dinámicas de otras materias, cabe resaltar que, en un diseño etnográfico, la descripción se basa en las observaciones que al investigador le resultan más importantes para mostrar la realidad que ha estudiado, es decir, aquellas que son útiles para contextualizar de manera más detallada los casos estudiados (Álvarez, 2008). En esa línea, la observación participante constató los discursos de las entrevistadas.
En términos similares, para la estudiante 1, el contenido de un curso que considera fundamental como Matemática presenta una continuidad de año en año, lo que no repercutirá en su desenvolvimiento posterior para tentar estudios superiores. Ella demanda una diversidad de temas que tengan concordancia con espacios más allá de la escuela a partir de la identificación de un mismo programa curricular año tras año.

La comparación entre la enseñanza del colegio y lo que se solicita para ingresar a la universidad muestra un abismo notorio que termina por influir en la valoración que estas estudiantes les dan a sus capacidades. Sienten encontrarse en un nivel básico del que buscan salir pensando en los resultados posteriores. El aprendizaje de Matemática tiene un propósito completamente instrumental e inmediato que es el ingreso a la universidad, pues, como muestra la tabla 1, la estudiante 2 del colegio de Surquillo está pensando estudiar Psicología o Derecho, carreras donde ese curso no es significativo. Contraponer lo básico a lo difícil, lo elemental a lo avanzado, en suma, la dicotomía colegio-universidad, revela que algunos conocimientos que se adquieren en 5to grado de educación secundaria se vuelven obsoletos porque no sirven a cuestiones prácticas como aprobar un examen de admisión.

La metodología mencionada anteriormente para que los adolescentes asimilen mejor Matemática no suma a los propósitos de las entrevistadas, quienes afirmaron que falta un poco y aún están en lo básico. Ellas priorizan más el fondo que la 
forma de dictar ese curso en la etapa preuniversitaria en la que se encuentran, donde la actualización de contenidos de la escuela puede garantizarles el ingreso a los estudios superiores, en los que están interesadas según los resultados de la tabla 1. Así, se evidencia que el componente pedagógico de la JEC no es bien visto por parte de los estudiantes porque los contenidos no les servirán para la universidad (Alcázar, 2016).

La modalidad de enseñanza de Matemática es definida por el Ministerio de Educación del Perú (MEP), así que su recepción por los estudiantes y su calificación como "básica" muestra los retos que tiene ese curso en 5to grado de educación secundaria. Como se ha podido observar, según la profesora, la JEC no solo ha incrementado las horas pedagógicas, sino que ha transformado la metodología de enseñanza de Matemática. El aprendizaje basado en problemas y saber leerlos son los ejes en los que se basa esta política educativa. Sin embargo, por parte de la profesora hay un reconocimiento de que todavía no se puede acoplar a una instancia universitaria.

En el colegio de SJM, se considera que los profesores que recién se han incorporado muestran más compromiso con la enseñanza, mientras que los antiguos no desarrollan metodologías para llegar a los estudiantes. La aceptación de los docentes está relacionada con la vocación profesional de los estudiantes. Por ejemplo, como se puede ver en la tabla 2, el estudiante 1 quiere estudiar Diseño Gráfico y el arte puede ser un componente fundamental para su carrera, pero en el colegio no encuentra la formación adecuada para su propósito profesional. Pese a decir que la mayoría de profesores no se está desempeñando como él espera, admite que el profesor nuevo de Matemática está dictando bien.

No solo la comparación entre los profesores nuevos y antiguos evidencia los cambios que ha tenido el colegio de SJM con la JEC. De acuerdo con el testimonio de una adolescente que estudia ahí desde el inicio de la secundaria, a lo largo de ese nivel la educación ha mejorado. Nuevamente, el curso de Matemática es relevante para los estudiantes. La diferencia es que en el colegio de SJM se afirma que está siendo bien enseñado. De esta manera, se encuentra que la apuesta de la JEC por Matemática tiene efectos distintos en un colegio de Surquillo y en otro de SJM. A los jóvenes de este último distrito parece servirles más esa área curricular que a los de Surquillo.

La interpretación de distintas circunstancias de la vida cotidiana utilizando el conocimiento adquirido en Matemática, objetivo que ha establecido la JEC y que fue confirmado con la observación participante, no le facilita la inserción a la universidad a una participante del estudio en el colegio de Surquillo. Por su parte, los escolares de SJM, que también apuntan a cursar estudios superiores, perciben que esa metodología ha contribuido a renovar sus aprendizajes.

Los resultados de la JEC no solo varían por colegio, sino también por grado. De acuerdo con la evidencia de Agüero (2016), los estudiantes de 2do grado de 
educación secundaria fueron los que más se beneficiaron con la mejora de sus aprendizajes de Matemática. En efecto, como señala la tabla 4, el uso del juego, la relación con la vida real y experimentos simples para explicar conceptos matemáticos pueden ser estrategias para enseñar mejor a otros grados del nivel secundario, pero no responden a las necesidades de un adolescente que se encuentra en el último año escolar.

El testimonio de una de las estudiantes del colegio de Surquillo indica que lo aprendido en Matemática no le favorecerá una trayectoria postsecundaria como ella espera. Así, se encuentra que el incremento de horas lectivas no ha influido positivamente en el caso de una adolescente que ha estudiado en ese centro educativo desde el nivel inicial $y$, por ende, ha experimentado el cambio de la JER a la JEC. Esto comprueba que los conocimientos del colegio no son suficientes para aprobar los exámenes de ingreso a la educación superior (Guerrero et al., 2016).

El análisis comparativo de los hallazgos en ambos colegios permite observar que en SJM la estudiante 3 valora el hecho de estudiar a tiempo completo. La cantidad de horas pedagógicas define la calidad de su escuela. Asimismo, la autoridad de los profesores y la disciplina son razones que la llevan a estar conforme con su formación bajo el modelo de la JEC. Por su parte, ninguna de las entrevistadas en la escuela de Surquillo se refirió a los beneficios de estudiar con una jornada escolar extendida.
Cabe resaltar que, durante el trabajo de campo, la estudiante 3 era nueva en el colegio de SJM. Llegó en 5to grado de educación secundaria procedente de una institución educativa con la JER en VMT. La comparación con su centro de estudios anterior le permite aceptar que su nueva escuela es mejor. Este hallazgo confirma que estudiar en un colegio con la JEC es motivo de distinción para una joven que vive en Lima Sur porque recibe más horas de enseñanza.

Pese a que la estudiante 3 se encarga de su hermano menor porque sus padres permanecen muchas horas al día fuera de casa por motivos laborales, no refirió ningún tipo de problema entre sus deberes en el hogar y el horario escolar extendido. Se observa que la JEC no tiene un efecto desfavorable en las actividades cotidianas de esta adolescente $\mathrm{y}$, por el contrario, reconoce que esa modalidad de estudios ha mejorado su aprendizaje.

Como punto en común, dos alumnas, una por cada centro educativo, destacaron la enseñanza de áreas curriculares como Formación Ciudadana y Cívica, y Persona, Familia y Relaciones Humanas como un motivo para definir sus perfiles vocacionales. Estas materias no han tenido un incremento en las horas lectivas con la JEC, lo que evidencia que esta política educativa no va en dirección con las apuestas profesionales de algunos estudiantes.

Por otra parte, más allá del objetivo del presente artículo, mostrar los efectos de la JEC sobre el aprendizaje de los estudiantes, los resultados dan cuenta que 
los egresados de estos colegios tienen expectativas de profesionalización, pero a la vez reconocen que primero tienen que trabajar. Así, se puede ver que también son conscientes de las influencias externas que pueden afectar o no sus oportunidades educativas. En la misma perspectiva, Post (1987) encuentra que los escolares de 5to grado de educación secundaria pueden reflexionar más sobre su futuro y tener mayor conciencia sobre su realidad personal.

Cabe señalar que, para obtener cierta uniformidad en la investigación, lo más idóneo hubiera sido contar con la misma cantidad de información por centro educativo. Al tratarse de un enfoque etnográfico, cualquier instrumento pudo utilizarse para obtener los datos, sin que ello limite la interpretación de cada realidad. En esa línea, ya que no se realizó observación participante a Matemática ni se entrevistó al docente de esa asignatura en el colegio de SJM, las entrevistas a los escolares igual ofrecieron material para el análisis y así se pudo comparar sus testimonios con los de sus pares en Surquillo. Dado que el objetivo del artículo es conocer los efectos de la JEC sobre el aprendizaje de los alumnos, sus percepciones son la prioridad.

\section{Conclusiones}

Los resultados generales obtenidos en este estudio permiten concluir que los efectos de la implementación de la JEC en dos escuelas públicas de Lima son heterogéneos y varían según el distrito de procedencia y las aspiraciones de los estudiantes. Los datos encontrados constatan que, en el caso de Surquillo, existen tensiones entre el componente pedagógico de Matemática y las aspiraciones al finalizar la etapa escolar, como es la aprobación de un examen de admisión. Esto concuerda con la investigación de Guerrero et al. (2016), que refiere que los adolescentes perciben que los conocimientos adquiridos en el colegio no son apropiados para las evaluaciones de acceso a la formación superior. Por su parte, es de notar que en SJM se reconoce que se ha enriquecido la enseñanza de ese curso y que la JEC es mejor que la jornada regular por la disciplina y el aumento de horas lectivas.

Asimismo, algunos testimonios evidenciaron que, si bien la JEC aumenta las aspiraciones educativas de sus usuarios (Sánchez y Favara, 2019), el incremento de horas lectivas no influye favorablemente en las trayectorias postsecundarias de los adolescentes. En relación a los instrumentos, las entrevistas confirmaron que para dos estudiantes del colegio de Surquillo la enseñanza de Matemática es básica y no se sienten preparadas para rendir satisfactoriamente un examen de admisión, y la observación participante mostró las características de la metodología de ese curso para explicar por qué no está siendo conveniente para algunos escolares de 5to grado de educación secundaria. Estos resultados comprueban la validez de un estudio etnográfico y sus componentes (Guber, 2001; Serra, 2004; Álvarez, 2008; 2011; Restrepo, 2016), así como dan cuenta de su pertinencia para analizar los efectos de la JEC sobre el aprendizaje de los alumnos. 
La JEC se ha propuesto responder a los intereses y las necesidades de los educandos, pero para ello se necesita una clasificación de la metodología de enseñanza según los grados y los tipos de estudiantes. Como señala Agüero (2016), los escolares de 2do grado de educación secundaria mejoraron su rendimiento en Matemática. Sin embargo, la conclusión de este estudio es que el aprendizaje basado en problemas, que ahora con la JEC se ha implementado en ese curso, no siempre tiene el mismo efecto favorable en un estudiante de 5to grado de educación secundaria, puesto que demanda contenidos que se consideran en los exámenes de admisión para las universidades.

Los resultados de esta investigación sirven para analizar los efectos del incremento de las horas lectivas en la transición del colegio al instituto de formación superior, a la universidad o al trabajo. Para una adolescente de la escuela de Surquillo, ubicada en un contexto urbano, teniendo en cuenta sus expectativas de profesionalización, más horas no representan una ventaja, pero pueden registrarse otros casos en los que sí, de acuerdo con el género y el colegio de procedencia. Así, en el colegio de SJM, dos estudiantes coinciden en que la enseñanza de Matemática ha mejorado.

Más allá de que sea un objetivo o no de la JEC dotar a los estudiantes de los medios necesarios para su incorporación a la vida social, a lo que sí apunta esta política es a promover el cierre de brechas y la equidad educativa en el Perú. Los resultados de esta investigación demuestran que, al menos con relación a 5 to grado de educación secundaria, los efectos positivos y negativos se dan según los contextos de las escuelas y las particularidades de los estudiantes. En términos similares, Alcázar (2016) encontró que los logros de la JEC son heterogéneos dependiendo del contexto donde se implemente. Además, los resultados obtenidos son congruentes con el reporte de Herrera et al. (2002), que señala que los centros educativos y su cultura escolar influyen en los dominios de vida de los adolescentes.

En caso haya un retorno a las clases presenciales mientras continúe el estado de emergencia sanitaria por COVID-19, se abren posibilidades para la reformulación de la JEC. Si se dispone que los estudiantes asistan a sus centros educativos por turnos, el incremento de horas lectivas para ciertos cursos, principal característica de la JEC, será inviable. En efecto, el futuro de esta política educativa es incierto, lo que permite elaborar propuestas para un nuevo horario escolar sin perjuicio de los intereses y las necesidades de los estudiantes.

Así como la JEC ha establecido un incremento horario de las áreas curriculares de Inglés, Comunicación, Matemática y EPT en todos los grados de secundaria, una recomendación de política puede ser que en 5to grado de educación secundaria esto varíe e incluya materias y conocimientos que se abordan en los exámenes de admisión. Por lo tanto, se propone un trabajo conjunto entre los docentes de aquellas asignaturas que se tienen en cuenta en ese tipo de 
evaluaciones, para que refuercen los contenidos que se incluyen en ellas. Estas sesiones se podrían trabajar en varios bloques a la semana, dentro del horario que estipula la JEC.

Es necesario considerar la demanda de contenido útil para el adolescente en su proyección para la universidad (Alcázar, 2016). Para lograrlo, si Matemática está entre los cursos que contarán con 10 horas lectivas a la semana en el largo plazo, su distribución puede ser 5 horas de enseñanza bajo el enfoque por competencias y 5 horas para el desarrollo de los temas presentes en los exámenes de admisión, en el caso de las secciones de 5to grado de educación secundaria.

En el marco de los proyectos piloto anteriores a la JEC, se organizaban talleres extracurriculares, como Periodismo, Cosmetología y Aplicaciones Tecnológicas. En esa línea, es recomendable que experiencias de este tipo se repitan con más profesiones $\mathrm{u}$ oficios a fin de que los escolares sepan de qué tratan las carreras que van a estudiar. Una incursión inicial en sus vocaciones puede ser decisiva para sus trayectorias futuras.

\section{Referencias}

Agüero, J. (2016). Evaluación de impacto de la Jornada Escolar Completa. Documento técnico. GRADE $\mathrm{y}$ FORGE. http://www.grade.org.pe/forge/descargas/Evaluaci\%C3\%B3n\%20de\%20impacto\% 20de\%20la\%20jornada\%20escolar\%20completa.pdf

Alanoca, R. (2016). Nivel de conocimiento acerca del modelo de servicio educativo Jornada Escolar Completa de directores de las instituciones educativas secundarias públicas de la región Puno [Tesis de doctorado, Universidad Nacional del Altiplano]. Repositorio Institucional Universidad Nacional del Altiplano.

Alcázar, L. (2016). Evaluación del diseño y proceso de implementación del modelo de Jornada Escolar Completa (JEC) para educación secundaria a nivel nacional. Informe final. GRADE y FORGE. http://www.grade.org.pe/forge/descargas/Informe\%20Final\%20\%20Evaluaci\%C3\%B3n\%20JEC\%20-\%20Lorena\%20Alcazar.pdf

Álvarez, C. (2008). La etnografía como modelo de investigación en educación. Gazeta de Antropología, 24 (1), 1-15. https://dx.doi.org/10.30827/Digibug.6998

Álvarez, C. (2011). El interés de la etnografía escolar en la investigación educativa. Estudios Pedagógicos, 37 (2), 267-279. https://doi.org/10.4067/S0718-07052011000200016

Alvira, F. (1983). Perspectiva Cualitativa-Perspectiva Cuantitativa en la metodología sociológica. Revista Española de Investigaciones Sociológicas, 22, 53-75. http://www.reis.cis.es/REIS/PDF/REIS 022 05.pdf 
Ames, P. y Rojas, V. (2011). "Podemos aprender mejor": percepciones de los niños, niñas y adolescentes peruanos sobre su educación. Un estudio en Lima, Piura, Ucayali y Ayacucho. Consejo Nacional de Educación y Fundación SM.

Ames, P., Rojas, V. \& Portugal, T. (2010). Metodologías para la investigación con niños. Lecciones aprendidas, desafíos y propuestas desde la experiencia de Niños del Milenio en Perú. GRADE.

Appadurai, A. (2004). The capacity to aspire. En Cultural Politics in a global age (pp. 59-84). One World.

Banco Mundial (2016). Ninis en América Latina. 20 millones de jóvenes en busca de oportunidades.

Benavides, M., Olivera, I. \& Mena, M. (2006). De papás y mamás a hijos e hijas: las aspiraciones sobre el futuro y el rol de las familias en las actividades escolares en el Perú rural. En Los desafíos de la escolaridad en el Perú. Estudios sobre los procesos pedagógicos, los saberes previos y el rol de las familias (pp. 157-214). GRADE.

Bernal, R., Pulido, X., Sánchez, F. \& Sánchez, L. (2018). Decisiones de vida de los jóvenes en Bogotá: ¿pobreza, habilidades o comportamientos de riesgo? En Millennials en América Latina y el Caribe: ¿trabajar o estudiar? (pp. 167-200). Banco Interamericano de Desarrollo.

Blasco, T. y Otero, L. (2008). Técnicas conversacionales para la recogida de datos en investigación cualitativa: la entrevista (1). Nure Investigación, 33, 1-5. https://www.nureinvestigacion.es/0JS/index.php/nure/article/view/408/399

Ccopa, S. (2018). Nivel de satisfacción laboral del modelo de servicio educativo Jornada Escolar Completa del personal de la Gran Unidad Escolar San Juan Bosco de Puno [Tesis de maestría, Universidad Nacional del Altiplano]. Repositorio Institucional Universidad Nacional del Altiplano.

Contreras, M., Novella, R., Nun, E. \& Repetto, A. (2018). Millennials en Chile: ¿estudiar, trabajar, ser nini? En Millennials en América Latina y el Caribe: ¿trabajar o estudiar? (pp. 122165). Banco Interamericano de Desarrollo.

Eguren, M. y de Belaunde, C. (2012). De lo colectivo a lo individual: las relaciones Estado-sociedad según la escuela peruana a inicios del nuevo siglo. IEP.

Favara, M. y Sánchez, A. (2019). Consequences of Teenage Childbearing in Peru: Is the Extended School-day Reform an Effective Policy Instrument to Prevent Teenage Pregnancy? Documento de trabajo № 185. Niños del Milenio. https://www.younglives.org.uk/sites/www.younglives.org.uk/files/YL-WP185.pdf 
Feliciano, G. (2017). El clima organizacional y el desempeño docente en la participación de la gestión en las instituciones educativas con Jornada Escolar Completa de la provincia de Arequipa - UGEL Norte, 2016 [Tesis de doctorado, Universidad Nacional de San Agustín de Arequipa]. Repositorio Institucional Universidad Nacional de San Agustín de Arequipa.

Gantu, I. (2018). Efectos sociales de la política educativa de incremento de la jornada escolar, desde la percepción de los miembros de la comunidad educativa [Tesis de maestría, Universidad Nacional Mayor de San Marcos]. Repositorio Institucional Universidad Nacional Mayor de San Marcos.

Guerrero, G. (2014). "Yo sé que va a ir más allá, va a continuar estudiando": expectativas educativas de estudiantes, padres y docentes en zonas urbanas y rurales del Perú. Documento de investigación № 74. GRADE. https://www.grade.org.pe/wpcontent/uploads/ddt74.pdf

Guerrero, G., Sugimaru, C., Cussianovich, A., De Fraine, B. \& Cueto, S. (2016). Education Aspirations among Young People in Peru and their Perceptions of Barriers to Higher Education. Documento de Trabajo № 148. Niños del Milenio. https://www.grade.org.pe/wp-content/uploads/YL WP148-Guerero Educationaspirations-in-Peru.pdf

González, N., Eguren, M. \& de Belaunde, C. (2017). Desde el aula: una aproximación a las prácticas pedagógicas del maestro peruano. IEP.

Guber, R. (2001). La etnografía. Método, campo y reflexividad. Grupo Editorial Norma.

Herrera, D., Lagrou, L. \& Lens, W. (2002). Inserción social en adolescentes: Un estudio sociopsicológico.

Persona,

5 ,

167-190. https://doi.org/10.26439/persona2002.n005.875

Ibáñez, J. (1986). Perspectivas de la investigación social: el diseño en la perspectiva estructural. En El análisis de la realidad social. Métodos y técnicas de investigación (pp. 31-65). Alianza Editorial.

López, P. (2004). Población muestra y muestreo. Punto Cero, 09 (08), 69-74. http://www.scielo.org.bo/scielo.php?script=sci arttext\&pid=S181502762004000100012

Mamani, G. (2017). La percepción del padre de familia de la Jornada Escolar Completa de la institución educativa secundaria Tawantinsuyo de la provincia de Desaguadero 2015 [Tesis de licenciatura, Universidad Nacional del Altiplano]. Repositorio Institucional Universidad Nacional del Altiplano. 
Marcus, G. y Fischer, M. (1986). La consideración de la economía política histórico-mundial: comunidades cognoscibles en sistemas más vastos. En La antropología como crítica cultural. Un momento experimental en las ciencias humanas (pp. 123-168). Amorrortu Editores.

Montero, C., González, N., Belaúnde, C., Eguren, M. \& Uccelli, F. (2009). El Estado de la educación: estudios sobre políticas, programas y burocracias del sector. IEP.

Oliart, P. (2011). Vida universitaria y masculinidades mestizas. En Políticas educativas y la cultura del sistema escolar en el Perú. IEP.

Peralta, Y. (2018). Logros de aprendizaje en el área de Inglés en las instituciones educativas secundarias con modelo Jornada Escolar Completa y Jornada Escolar Regular de la provincia de Melgar - Puno 2017 [Tesis de segunda especialidad, Universidad Nacional del Altiplano]. Repositorio Institucional Universidad Nacional del Altiplano.

Portocarrero, G. y Oliart, P. (1989). El Perú desde la escuela. Instituto de Apoyo Agrario.

Post, D. (1987). Determinantes de las metas políticas entre los escolares cusqueños del último año de secundaria. Revista Peruana de Ciencias Sociales, 1, 173-197. Fomciencias.

Restrepo, E. (2016). Trabajo de campo. En Etnografía: alcances, técnicas y éticas (pp. 35-65). Envión Editores.

Rojas, V. y Cussianovich, A. (2013). Le va bien en la vida: cambios y permanencias en el bienestar subjetivo de un grupo de adolescentes en Perú. Documento de trabajo. GRADE y Niños del Milenio. http://www.grade.org.pe/upload/publicaciones/archivo/download/pubs/dt.pdf

Rojas, D. y Ramos, M. (2017). Comparación del nivel de estrés académico en estudiantes de colegios con Jornada Escolar Completa y Jornada Escolar Regular de Cajamarca 2017 [Tesis de licenciatura, Universidad Privada Antonio Guillermo Urrelo]. Repositorio Institucional Universidad Privada Antonio Guillermo Urrelo.

Ruano, O. (2007). El trabajo de campo en investigación cualitativa (II). Nure Investigación, 29, 1-4. https://www.nureinvestigacion.es/OJS/index.php/nure/article/view/339/329

Serra, C. (2004). Etnografía escolar, etnografía de la educación. Revista de Educación, 334, 165176. http://www.educacionyfp.gob.es/dam/jcr:cd4cd01f-76cf-4601-add1$\underline{41 \mathrm{~b} 28 \mathrm{e} 0 \mathrm{e} 08 \mathrm{a} 6 / \mathrm{re} 33411-p d f . p d f}$

Villegas, M. (2019). Niveles de lectura en estudiantes de segundo grado de secundaria con Jornada Escolar Completa y Jornada Escolar Regular en La Región Madre de Dios - 2018 [Tesis de maestría, Universidad César Vallejo]. Repositorio Institucional Universidad César Vallejo. 
Yana, M. y Adco, H. (2018). Acompañamiento pedagógico y el rol docente en Jornada Escolar Completa: Caso instituciones educativas Santa Rosa y Salesianos de San Juan Bosco Puno Perú. Revista de Investigaciones Altoandinas, 20 (1), 137-148. Universidad Nacional del Altiplano. 